AperTO - Archivio Istituzionale Open Access dell'Università di Torino

\title{
M2td: Multi-task tensor decomposition for sparse ensemble simulations
}

\section{This is the author's manuscript}

Original Citation:

\section{Availability:}

This version is available http://hdl.handle.net/2318/1782486

since 2021-03-24T18:16:47Z

Publisher:

Institute of Electrical and Electronics Engineers Inc.

Published version:

DOI:10.1109/ICDE.2018.00106

Terms of use:

Open Access

Anyone can freely access the full text of works made available as "Open Access". Works made available under a Creative Commons license can be used according to the terms and conditions of said license. Use of all other works requires consent of the right holder (author or publisher) if not exempted from copyright protection by the applicable law. 


\section{M2TD: Multi-Task Tensor Decomposition for Sparse Ensemble Simulations}

\author{
Xinsheng Li \\ Arizona State University \\ Tempe, AZ 85287, USA \\ Email: 1xinshen@asu.edu
}

\author{
K. Selçuk Candan \\ Arizona State University \\ Tempe, AZ 85287, USA \\ Email:candan@asu.edu
}

\author{
Maria Luisa Sapino \\ University of Torino \\ I-10149 Torino, Italy \\ Email:marialuisa.sapino@unito.it
}

\begin{abstract}
Data- and model-driven computer simulations are increasingly critical in many application domains. These simulations may track 10s or 100s of parameters, affected by complex inter-dependent dynamic processes. Moreover, decision makers usually need to run large simulation ensembles, containing 1000s of simulations. In this paper, we rely on a tensor-based framework to represent and analyze patterns in large simulation ensemble data sets to obtain a high-level understanding of the dynamic processes implied by a given ensemble of simulations. We, further, note that the inherent sparsity of the simulation ensembles (relative to the space of potential simulations one can run) constitutes a significant problem in discovering these underlying patterns. To address this challenge, we propose a partition-stitch sampling scheme, which divides the parameter space into subspaces to collect several lower modal ensembles, and complement this with a novel Multi-Task Tensor Decomposition (M2TD), technique which helps effectively and efficiently stitch these subensembles back. Experiments showed that, for a given budget of simulations, the proposed structured sampling scheme leads to significantly better overall accuracy relative to traditional sampling approaches, even when the user does not have perfect information to help guide the structured partitioning process.
\end{abstract}

\section{INTRODUCTION}

Data- and model-driven computer simulations are increasingly critical in many application domains. For example, for predicting geo-temporal evolution of epidemics and assessing the impact of interventions, experts often rely on epidemic spread simulation software, such as STEM [6]. Simulationbased decision making, however, introduces several fundamental data challenges [23], [28]:

- Many complex processes (such as disasters [4]) involve various distinct, yet inter-dependent, sub-processes. Consequently, in order to be useful, these simulations may track 100s of parameters, spanning multiple layers and spatial-temporal frames, affected by complex interdependent dynamic processes (Figure 1).

- Moreover, due to large number of unknowns, decision makers usually need to generate an ensemble of stochastic realizations, requiring $1000 \mathrm{~s}$ of individual simulation

Research is supported by NSF\#1318788 "Data Management for RealTime Data Driven Epidemic Spread Simulations", NSF\#1339835 "E-SDMS: Energy Simulation Data Management System Software", NSF\#1610282 "DataStorm: A Data Enabled System for End-to-End Disaster Planning and Response", NSF\#1633381 "BIGDATA: Discovering Context-Sensitive Impact in Complex Systems", and "FourCmodeling": EU-H2020 Marie Sklodowska-Curie grant agreement No 690817.

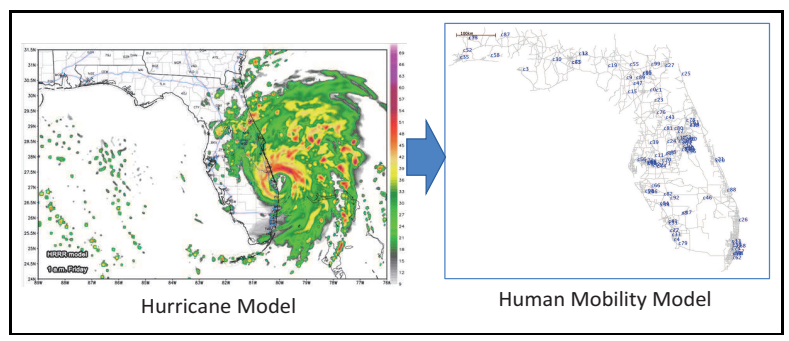

Fig. 1: Coupled simulation of a hurricane and human mobility

instances, each with different parameter settings corresponding to different, but plausible, scenarios.

Consequently, obtaining and interpreting simulation ensembles to generate actionable results present difficulties:

- Limited ensemble simulation budgets: Since complex, inter-dependent parameters affected by complex dynamic processes have to be taken into account, execution of simulation ensembles can be very costly. This leads to simulation budget constraints that limit the number of simulations one can include in an ensemble.

- Need for post-simulation data processing: Because of the complexities of key processes and the varying scales at which they operate, experts often lack the means to drive conclusions from these ensembles. This leads to the need for data analytics on simulation ensembles to discover broad, actionable patterns.

- Inherent data sparsity of simulation ensembles: While the size and complexity of a simulation ensemble can indeed tax decision makers, we note that a simulation ensemble is inherently sparse (relative to the space of potential simulations one could run), which constitutes a significant problem in simulation-based decision making. This leads to the following critical question: "Given a parameter space and a fixed simulation budget, which simulation instances we should include in the ensemble?"

\section{A. Tensor Representation of Simulation Ensembles}

In this paper, we propose a tensor-based framework to represent and analyze large simulation ensembles. Intuitively, the tensor model maps a multi-attribute schema to a multimodal array (where each potential tuple is a tensor cell). Consequently, we can map a given simulation ensemble onto 


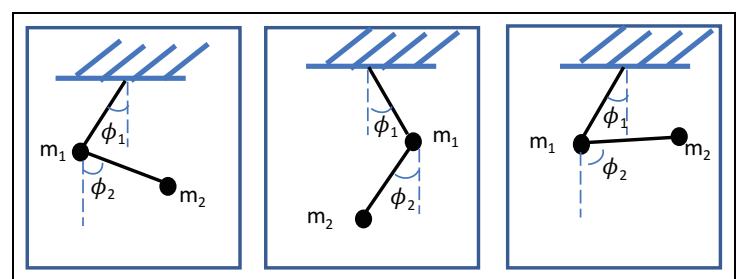

Fig. 2: States of a multi-pendulum system

a tensor such that each simulation parameter corresponds to a mode of a tensor and the non-null entries in the tensor represent results of the simulations we have executed.

Tensor decomposition [11], [32], [19] (which generalizes matrix decomposition to tensors) has been successfully used in various applications, such as social networks, sensor streams, and others [20]. Intuitively, the tensor decomposition process rewrites the given tensor in the form of a set of factor matrices (one for each mode of the input tensor) and a core matrix (which, intuitively, describes the spectral structure of the given tensor). As such, tensor decomposition has also been used for the analysis of dynamical systems: [29] proposed a tensorbased model for time series and [18] proposed a dynamic mode decomposition (DMD) scheme for the analysis of the behavior of complex dynamical systems.

\section{B. Inherent Sparsity of Ensembles}

While, as discussed above, tensors have been successfully used for understanding dynamic systems, we note that when the data is sparse, tensor decomposition is less effective in extracting meaningful information - which is a significant challenge when we are attempting to learn about dynamic processes through an inherently sparse ensemble of simulations. To see why, note that as the number of input parameters of a simulation increases, the number of potential situations one can simulate increases exponentially. Consider for example, the simple dynamical system, double equal-length pendulum, depicted in Figure 2: in this system there are five parameters that one can control: (a) the initial angle of the first pendulum $\phi_{1}$, (b) the initial angle of the second pendulum $\phi_{2}$, (c) the weight of the first bob $m_{1}$, (d) the weight of the second bob $m_{2}$, and (b) the gravity, $g$. For each combination of parameter values, the system can be viewed as a two-variate time series consisting of the angles of the pendulums at each time step. It is easy to see that the number of potential simulations of this double equal-length pendulum system is a function of the resolution of each of these four parameters - if we simply assume that for each parameter we consider, say, 20 distinct values, this would lead to $20^{5}=3200000$ possible simulations to potentially consider. Assuming that we have a simulation budget, $B=1000$, this would lead to a simulation density of only $1000 / 3200000 \sim 0.0003125$. Therefore, even for a relatively small number of parameters, any realistic simulation budget is likely to be much smaller than the possible space of all simulations - consequently, the naive approach of randomly sampling the simulation space is likely to lead to sparse tensors that are difficult to accurately analyze.

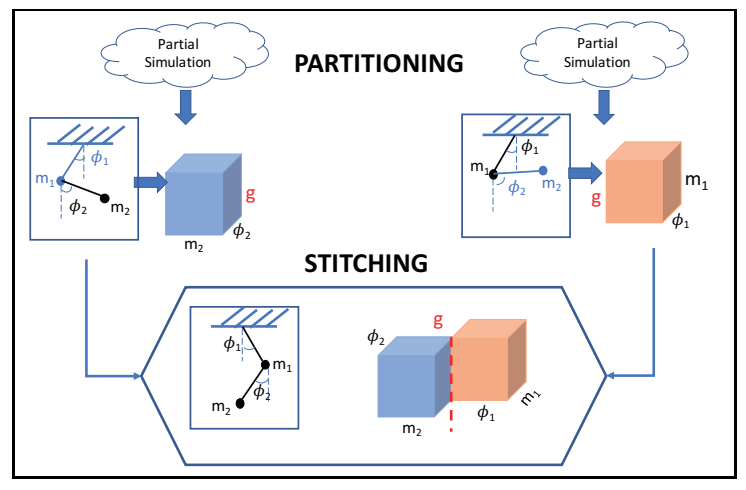

Fig. 3: Partition-Stitch sampling

\section{Contribution 1: Density Boosting Partition-Stitch Sampling}

In this paper, we propose an alternative ensemble creation strategy, which we refer to as the partition-stitch sampling (Figure 3): given an $N$-parameter simulation and an ensemble budget of $B$, instead of randomly allocating the $B$ samples in the $N$-dimensional parameter space, we partition the simulation space into $\sim N / 2$ dimensional sub-spaces and allocate $B / 2$ simulations for each sub-space: note that, since the number of possible simulations for each sub-space reduced exponentially (in the number of excluded parameters), this corresponds to an exponential increase in the density of the samples for each sub-space: let us re-consider the double equal-length pendulum system in Figure 2: instead of considering the original 5-parameter system, we can divide the simulation space into simulations for two 3-parameter systems:

- System 1: In this system, we are allowed to vary the initial angle, $\phi_{1}$, and weight, $m_{1}$, of the first pendulum as well as the gravity, $g$; but the initial angle, $\phi_{2}$, and weight, $m_{2}$, of the second pendulum are fixed.

- System 2: In the second system, we can $\overline{\text { vary }}$ the initial angle, $\phi_{2}$, and weight, $m_{2}$, of the second pendulum as well as the gravity, $g$; in this case, the initial angle, $\phi_{1}$, and weight, $m_{1}$, of the first pendulum are fixed.

Note that neither of the two systems are perfect representations of the overall behavior of the whole system as, in both cases, two out of the five parameters are fixed to some default values. However, the simulation densities of both systems are now much higher than the simulation density of the original system: using the numbers considered earlier, each sub-system has 3 parameters with 20 distinct values, leading to a parameter space of $20^{3}=8000$ simulations. If we allocate $500(=1000 / 2)$ simulations to each sub-space, this leads to a simulation density of $500 / 8000=0.0625$, which is 200 time denser than the original simulation space. There, however, remain several important questions:

- The first important question is "How do we stitch back the results obtained from the individual sub-spaces?"

Here we may have several alternatives: In the simplest alternative, all the simulations from the two systems can be unioned into a single 5-mode tensor and this 5-mode tensor can be decomposed for analysis. This is potentially very expensive as 
the decomposition cost often increases exponentially with the number of modes of the input tensor [17], [22]. We will also see that, once unioned into a single tensor, the overall density is still low and the accuracy gains will be very limited.

Instead, we will present a join-based scheme to increase the effective density of the ensemble. In particular, we will present two approaches (join stitching and zero-join stitching) to combine simulation results form the sub-systems and experimentally validate the effectiveness of these schemes. Several questions, however, remain:

- How do we select the parameter to be shared across the two sub-spaces?: We experimentally verify that the significant gains in accuracy due to the increase in simulation densities of the sub-systems reduces the need to be particularly careful in selecting the shared parameter.

- What about the fact that both partial systems use some default values to fix some of the parameters? Doesn't this negatively affect accuracy? We will see that the gains obtained in accuracy due to the significant jump in simulation densities will overcome any disadvantages associated with fixing some of the parameters.

- If we are joining the sub-ensembles back to the original $N$-parameter space, wouldn't this negatively effect the tensor decomposition cost? If done naively, yes; and we discuss this in the next sub-section.

\section{Contribution 2: Multi-Task Tensor Decomposition (M2TD)}

Naively joining the sub-ensembles would map the simulations back to an $N$-modal tensor and this would exponentially increase the tensor decomposition time. Instead, in this paper, we propose a novel Multi-Task Tensor Decomposition (M2TD) scheme, which reduces the computational complexity of high-order tensor decomposition by (a) first cheaply decomposing the low-order partial tensors and (b) intelligently stitching back the decompositions of these partial tensors to obtain the decomposition for the whole system. Intuitively, M2TD leverages partial and imperfect simulation-based knowledge from the resulting partial dynamical systems to obtain a global view of the complex process being simulated. In this paper, we study alternative ways one can stitch the tensor decompositions and propose an M2TD - SELECT that provides better accuracy than the alternatives.

\section{E. Organization of the Paper}

This paper is organized as follows: In the next section, we present the related work. Section III presents the relevant notations and the background. Section IV presents several conventional solutions to the problem and outlines their weaknesses. Section V describes the proposed partitionstitch sampling technique supported with a novel multi-task tensor decomposition approach (Section VI). Section VII experimentally evaluates the effectiveness of the M2TD and its alternative implementations. Experiments show that M2TD indeed improves the decomposition accuracy of high order tensors and handles much larger datasets than the current state of the art. We conclude the paper in Section VIII.

\section{RELATED WORK}

\section{A. Simulation Design}

Ensemble simulations are increasingly critical in many application domains [28], [30]. Yet, (a) designing an ensemble that appropriately covers the input parameter space [9] and (b) interpreting the simulations in the ensemble [23], [28] are not trivial.

Work in this area, primarily focused on the first problem, which is often handled through single- or multiple-run replications [26]: in single-run replication, simulation instances are allocated incrementally, at each step evaluating the performance and deciding the next simulation to run; in multiple-run replication, the parameter space is sampled simultaneously, resulting in multiple-shorter runs. A long line of work in the area is, then, focused on the development of performance estimators and experiment design strategies [10], [15]. More recently, budget constraints and costs of simulations are being taken into account in simulation instance selection [25]. In this paper, however, we note that the second problem is as important as the first one, and therefore one has to consider the two problems of designing ensemble simulations under budget constraints and interpreting the results - we therefore, propose, a tensor-based framework for ensemble simulations and present a partition-stitch strategy to effectively increase the ensemble density to provide more accurate tensor-based analysis of a given ensemble.

\section{B. Tensors and Scalable Tensor Decomposition}

As discussed earlier, tensor decomposition (such as CP [11] and Tucker [32]) is commonly used for analyzing multidimensional data [20]. Yet, the tensor decomposition process is subject to several major challenges: decomposition algorithms have high computational costs and, in particular, incur large memory overheads (also known as the intermediary data blowup problem) and, thus, basic algorithms and naive implementations are not suitable for large problems.

There are two widely used toolboxes for tensor manipulation: the Tensor Toolbox for Matlab [8] (for sparse tensors) and $N$-way Toolbox for Matlab [7] (for dense tensors). Parallel implementations, such as GridParafac [27], GigaTensor [16], HaTen2 [14], TensorDB [17], [22], [21], were proposed to deal with the high computational cost of the task. [31] proposes $\mathrm{MACH}$, a randomized algorithm that speeds up the Tucker decomposition while providing accuracy guarantees. In [24], authors propose PARCUBE, a sampling based, parallel and sparsity promoting, approximate PARAFAC decomposition scheme. Scalability is achieved through sketching of the tensor (using biased sampling) and parallelization of the decomposition operations onto the resulting sketches. TensorDB [17], [22], [12] leverages a block-based framework to store and retrieve data, extends array operations to tensor operation, and introduces optimization schemes for in-database tensor decomposition. HaTen2 [14] focuses on sparse tensors and presents a scalable tensor decomposition suite on a MapReduce framework. SCOUT [13] is a recent coupled matrixtensor factorization framework, also built on MapReduce. 


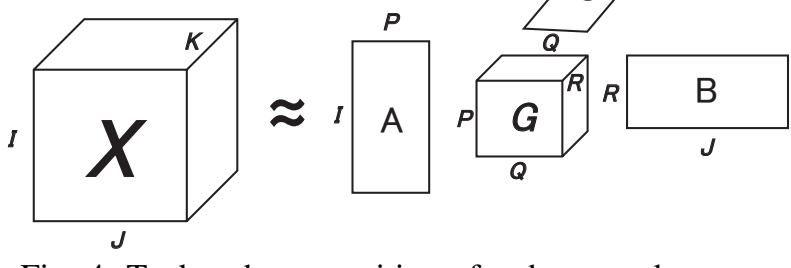

Fig. 4: Tucker decomposition of a three-mode tensor

\section{BACKGROUND AND NOTATIONS}

\section{A. Tensors}

The tensor model maps a multi-attribute schema into an $N$-modal array. More formally, let $I^{j}$ denote the number of distinct values that the $j^{\text {th }}$ attribute (or $j^{\text {th }}$ mode) can take. The tensor $\mathcal{X}$ is then an $N$ mode array such that $\mathcal{X} \in \mathbb{R}^{I_{1} \times I_{2} \times \ldots \times I_{N}}$. Intuitively, the modes of the tensor represent different factors that impact an observation and the value that the tensor records for a given cell corresponds to an observation for a specific combination of factor instances.

\section{B. Tensor Decomposition}

The two most popular tensor decomposition algorithms are the Tucker [32] and the CANDECOMP/PARAFAC (CP) [11] decompositions. In this paper, we focus on the Tucker decomposition of simulation ensemble tensors. Intuitively, the Tucker decomposition generalizes singular value matrix decomposition (SVD) to higher-dimensional data (Figure 4). Given a tensor $\mathcal{X}$, Tucker decomposition factorizes the tensor into factor matrices with different number of rows, which are referred to as the rank of the decomposition. For the simplicity of the discussion, let us consider a 3-mode tensor $\mathcal{X} \in \mathbb{R}^{I \times J \times K}$. Tucker decomposition would decompose $\mathcal{X}$ into three matrices $\mathbf{A}, \mathbf{B}, \mathbf{C}$ and one core dense tensor $\mathbf{g}$, such that

$\mathcal{X} \approx \tilde{\mathcal{X}}=\mathbf{g} \times{ }_{1} \mathbf{A} \times{ }_{2} \mathbf{B} \times{ }_{3} \mathbf{C} \equiv \sum_{p=1}^{P} \sum_{q=1}^{Q} \sum_{r=1}^{R} g_{p q r} a_{p} \circ b_{q} \circ c_{r}$,

where $\mathbf{A} \in \mathbb{R}^{I \times P}, \mathbf{B} \in \mathbb{R}^{J \times Q}, \mathbf{C} \in \mathbb{R}^{K \times R}$, are the factor matrices and can be treated as the principal components in each mode. The (dense) core tensor, $\mathrm{g} \in \mathbb{R}^{P \times Q \times R}$, indicates the strength of interactions among different components of the factor matrices.

It is important to note that tensors very rarely have exact Tucker decompositions. In almost all cases, the new tensor $\tilde{\mathcal{X}}$ obtained by recomposing the factor matrices $\mathbf{A}, \mathbf{B}, \mathbf{C}$ and core tensor $\mathbf{g}$ is often different from the input tensor, $\mathcal{X}$. The accuracy of the decomposition is often measured by considering the Frobenius norm of the difference tensor.

More generally, given an $N$-mode tensor, $\mathcal{X} \in \mathbb{R}^{I_{1} \times I_{2} \times \ldots \times I_{N}}$, and $N$ target rank values, $r_{1}$ through $r_{N}$, the corresponding Tucker decomposition is $\left[\mathcal{G}, \mathbf{U}^{(\mathbf{1})}, \mathbf{U}^{(\mathbf{2})}, \mathbf{U}^{(\mathbf{3})}, \ldots, \mathbf{U}^{(\mathbf{N})}\right]$, such that

$$
\tilde{\mathcal{X}}=\mathcal{G} \times{ }_{1} \mathbf{U}^{(1)} \times{ }_{2} \mathbf{U}^{(2)} \times{ }_{3} \mathbf{U}^{(3)} \ldots \times_{N} \mathbf{U}^{(\mathbf{N})} \approx \mathcal{X} .
$$

Here, $\mathbf{U}^{(\mathbf{i})}$ are the $N$ factor matrices and $\mathcal{G}$ is an $r_{1} \times \ldots \times r_{N}$ dimensional core tensor.

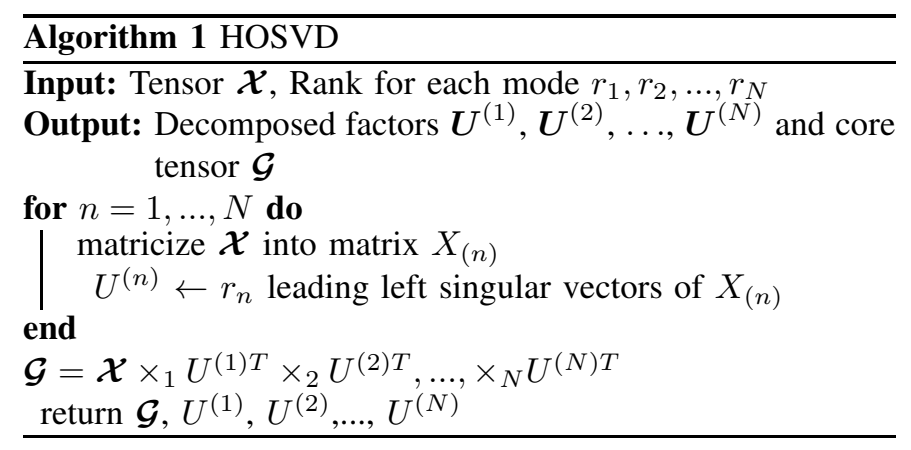

1) HOSVD Decomposition [20] : Algorithm 1 illustrates the HOSVD algorithm for Tucker decomposition of a given $N$-mode tensor, $\mathcal{X}$ for target rank values, $r_{1}$ through $r_{N}$. For each mode of the tensor, HOSVD matricizes (flattens) the high-order tensor $\mathcal{X}$ into a matrix. Then this matrix is decomposed (using SVD) to obtain the left eigenvectors and these are packed into a factor matrix for the corresponding mode. Finally, the core tensor is recovered from the original tensor and the $N$ factor matrices obtained as described.

\section{Tensor Representation of a Complex System}

Let us be given a complex dynamic system, $S$, with $N$ input parameters, such that the $i^{\text {th }}$ input parameter can take $I_{i}$ distinct values. For simplicity of the discussion, let us further assume that for each input parameter combination $\left\langle v_{1}, \ldots v_{N}\right\rangle$, the complex dynamic system $S$ generates a single value $S\left(v_{1}, \ldots, v_{n}\right)$. Let, further, $Y$ be the set of all simulations of the system $S$ one can execute and the corresponding results; i.e., $Y=\left\{y_{i}=\left\langle\left\langle v_{i, 1}, \ldots, v_{i, N}\right\rangle, S\left(v_{i, 1}, \ldots, v_{i, N}\right)\right\rangle \| 1 \leq i \leq\right.$ $\left.I_{1} \times I_{2} \times \ldots \times I_{N}\right\}$. It is easy to see that $Y$ can be encoded as a tensor $\mathcal{Y} \in \mathbb{R}^{I_{1} \times I_{2} \times \ldots \times I_{N}}$, where for all $y_{i} \in Y$, the tensor cell $\mathcal{Y}\left(v_{i, 1}, \ldots, v_{i, N}\right)$ has the value $S\left(v_{i, 1}, \ldots, v_{i, n}\right)$.

\section{Tensor Representation of a Simulation Ensemble}

The number, $I_{1} \times \ldots \times I_{N}$, of simulations of the system, $S$, one can run can be very large. Instead, as discussed in the introduction, we often run a much smaller subset (or ensemble) of the simulations to get an idea about $S$. Given an ensemble of $B \ll I_{2} \times \ldots \times I_{N}$ simulations, let $X$ be the set of simulations that have been selected to be executed as well as the corresponding system outputs; i.e., $X=\left\{x_{i}=\right.$ $\left.\left\langle\left\langle v_{i, 1}, \ldots, v_{i, N}\right\rangle, S\left(v_{i, 1}, \ldots, v_{i, N}\right)\right\rangle \| 1 \leq i \leq B\right\}$. It is easy to see that $X$ can be encoded as a tensor $\mathcal{X} \in \mathbb{R}^{I_{1} \times I_{2} \times \ldots \times I_{N}}$, where for all $x_{i} \in X$, the tensor cell $\mathcal{X}\left(v_{i, 1}, \ldots, v_{i, N}\right)$ has the value $S\left(v_{i, 1}, \ldots, v_{i, N}\right)$ and all other cells have null values (indicating simulations that could potentially have been run, but have not been included in the ensemble). Since $B \ll I_{1} \times I_{2} \times \ldots \times I_{N}$, the tensor $\mathcal{X}$ is very sparse, meaning that there will be many more null-valued cells than the cells recording real-valued simulation results.

\section{E. Problem Definition}

Ideally, to study the system, $S$, we would construct a complete tensor $\mathcal{Y} \in \mathbb{R}^{I_{1} \times I_{2} \times \ldots \times I_{N}}$, and given target rank values, 


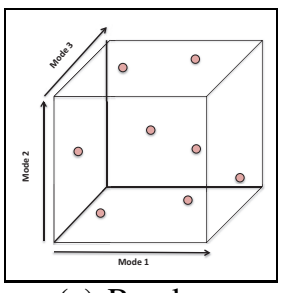

(a) Random

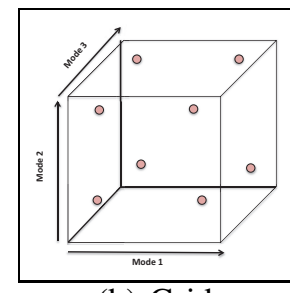

(b) Grid

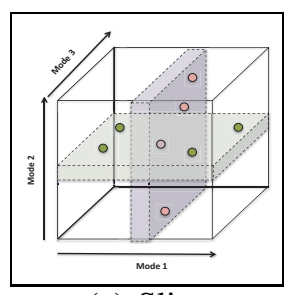

(c) Slice
Fig. 5: Conventional solutions for ensemble generation

$r_{1}$ through $r_{N}$, we would obtain its corresponding Tucker decomposition $\left[\mathcal{H}, \mathbf{V}^{(\mathbf{1})}, \mathbf{V}^{(\mathbf{2})}, \mathbf{V}^{(\mathbf{3})}, \ldots, \mathbf{V}^{(\mathbf{N})}\right]$, where

$$
\tilde{\mathcal{Y}}=\mathcal{H} \times{ }_{1} \mathbf{V}^{(\mathbf{1})} \times{ }_{2} \mathbf{V}^{(2)} \times{ }_{3} \mathbf{V}^{(3)} \ldots \times_{N} \mathbf{V}^{(\mathbf{N})} \approx \mathcal{Y} .
$$

However, this would be prohibitively costly:

- Firstly, this would require $I_{1} \times \ldots \times I_{N}$ simulations, which can be computationally overwhelming.

- Even if this many simulations can be obtained, the analysis of the resulting tensor may be prohibitively expensive. Instead, given a budget $B \ll I_{1} \times \ldots \times I_{N}$ of simulations, the problem is to identify a set, $X=\left\{x_{i}=\right.$ $\left.\left\langle\left\langle v_{i, 1}, \ldots, v_{i, N}\right\rangle, S\left(v_{i, 1}, \ldots, v_{i, N}\right)\right\rangle \| 1 \leq i \leq B\right\}$ of $B$ simulations to execute, such that the Tucker decomposition $\left[\mathcal{G}, \mathbf{U}^{(\mathbf{1})}, \mathbf{U}^{(\mathbf{2})}, \mathbf{U}^{(\mathbf{3})}, \ldots \mathbf{U}^{(\mathbf{N})}\right]$ of the corresponding tensor $\mathcal{X}$ has the following property:

$$
\tilde{\mathcal{X}}=\mathcal{G} \times{ }_{1} \mathbf{U}^{(1)} \times{ }_{2} \mathbf{U}^{(2)} \times_{3} \mathbf{U}^{(3)} \ldots \times_{N} \mathbf{U}^{(\mathbf{N})} \approx \mathcal{X},
$$

and the Frobenius norm, $\|\mathcal{Y}-\tilde{\mathcal{X}}\|_{F}$, of the difference (from the full simulation ensemble, $\mathcal{Y}$ ) is small.

\section{Conventional Ensemble Sampling Strategies}

\section{A. Strategy \#1: Random Sampling}

The first approach for creating a budget constrained ensemble of simulations for the system $S$ is to uniformly randomly sample $B \ll I_{1} \times \ldots \times I_{N}$ parameter value configurations in the parameter space and execute those $B$ randomly sampled simulations to obtain the ensemble, $X_{r s}$ (Figure 5(a)).

\section{B. Strategy \#2: Grid Sampling}

The second approach for creating a budgeted ensemble of simulations for $S$ is to sample $B$ parameter value configurations at positions defined by a regularly spaced grid and execute those $B$ sampled simulations to obtain the ensemble, $X_{g s}$ (Figure 5(b)).

\section{Strategy \#3: Slice Sampling}

As we can see from Figures 5(a) and (b), the major difference between random sampling and grid sampling is that in grid-based ensemble construction, the subsets of the selected simulation samples are aligned on vertical and horizontal directions (or slices) of the underlying tensor and these vertical and horizontal slices cover the tensor regularly. Alternatively, these slices and the samples within each slice can be randomly selected. Intuitively, each slice fixes one of the parameters, therefore, the samples within each slice are denser (whereas the density of the overall tensor remains the same). We refer to the resulting ensemble as $X_{s s}$.

\section{Partition-Stitch SAmpling}

The three alternatives presented in the previous section cover the underlying parameter space in different ways using the same number of simulation instances. Consequently, while the local sub-space densities may differ, the overall simulation density is identically low for all three cases.

In this section, we show that, while executing the same number $(B)$ of simulation instances as before, we can increase the effective simulation density of the ensemble by carefully partitioning the simulations to run into two groups and, then, by carefully stitching them, relying on shared information among these groups to transfer knowledge among them.

\section{A. Key Observation}

The key observation is that most complex processes can be partitioned such that, while each partition captures different sub-processes, these nevertheless relate to each other and, hence, reflect the footprints of the same underlying global pattern. Therefore, at least in theory, it should be possible to partition the given system $S$ into two sub-systems $S_{1}$ and $S_{2}$, and analyze them independently. Transferring what we independently learned from the analysis of $S_{1}$ and $S_{2}$ backand-forth, we should be able to gather information regarding the original global system, $S$. To leverage this observation, however, we need to answer two major questions: (a) "How do we partition the system, S, into two sub-systems?" and (b) "How do we stitch the outcomes of these two sub-systems, $S_{1}$ and $S_{2}$, back to learn about $S$ ?"

\section{B. PF-Partitioning of a Parameter Space}

It turns out that the answer to the first question is relatively straightforward: Given a system $S$ with $N$ input parameters, we will partition the system into two sub-systems $S_{1}$ and $S_{2}$, each with $\frac{N-k}{2}+k$ input parameters, such that

- the two systems share $k$ of their input parameters as pivot parameters, and

- for each system, the remaining $\frac{N-k}{2}$ parameters will be set to a default value, referred to as fixing constants.

We will refer to this as the Pivoted/Fixed (PF)-partitioning of a parameter space. Intuitively, $S_{1}$ and $S_{2}$ correspond to two constrained sub-spaces: they have lesser free parameters than the original system $S$ as each one is generated by fixing $\frac{N-k}{2}$ of the input parameters. Once the two sub-systems are obtained through PF-partitioning, we can then create two sets, $X_{1}$ and $X_{2}$, of ensembles (through random, grid, or slice sampling), each with $B / 2$ simulations - these simulations are created with common values for shared pivot parameters. Consequently, the pivot parameters can be used for stitching the two ensembles together. More formally, let $\rho_{1}, \ldots, \rho_{i}, \ldots \rho_{N}$ denote the $N$ input parameters of $S$, each with a domain with $I_{i}$ distinct values. Without loss of generality, we refer to

- $\rho_{1}$ through $\rho_{k}$ as the pivot parameters,

- we select $P \leq I_{1} \times \ldots \times I_{k}$ possible configurations for the pivot parameters for ensemble generation, 


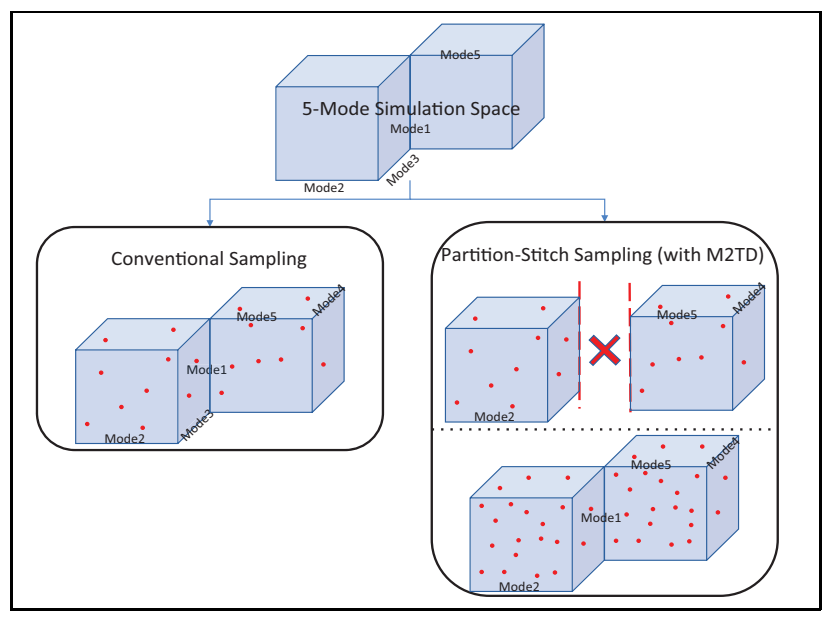

Fig. 6: Ensemble creation through PF-partitioning, followed by JE-stitching provides a higher effective density than the convention sampling of the original parameter space

- $\rho_{k+1}$ through $\rho_{k+(N-k) / 2}$ will serve as the free input parameters of system $S_{1}$ and fixed parameters of $S_{2}$,

- we select $E \leq I_{k+1} \times \ldots \times I_{k+(N-k) / 2}$ possible configurations for the free parameters for ensemble generation for system $S_{1}$,

- $\rho_{k+(N-k) / 2+1}$ through $\rho_{N}$ will serve as the free input parameters of system $S_{2}$ and fixed parameters of $S_{1}$.

- we select $E \leq I_{k+(N-k) / 2+1} \times \ldots \times I_{N}$ possible configurations for the free parameters for ensemble generation for system $S_{2}$.

Note that, given the input budget $B$, we have $P \times E=B / 2$. In the next sub-section, we discuss how to stitch these subensembles to increase the overall effective density.

\section{JE-Stitching}

As we mentioned above, the goal of the stitching process is to increase the effective density of the ensemble. JoinEnsemble (JE)-Stitching achieves this by joining or zerojoining the two sub-systems along the shared modes:

1) Join-based Stitching: Let $\mathcal{X}_{1}$ and $\mathcal{X}_{2}$ denote the two tensors representing the simulation ensembles, $X_{1}$ and $X_{2}$, for the two sub-systems $S_{1}\left(\rho_{1,1}, \ldots, \rho_{1, k+(N-k) / 2}\right)$ and $S_{2}\left(\rho_{2,1}, \ldots, \rho_{2, k+(N-k) / 2}\right)$, respectively. For simplicity, let the first $k$ parameters of both sub-systems denote the set of parameters shared between the two sub-systems. We construct a new join ensemble, $J$, as follows: for all pairs of simulations in the two ensembles that agree on the parameter values for the $k$ shared parameters (i.e., $\left.\left(\rho_{1,1}=\rho_{2,1}\right) \wedge \ldots \wedge\left(\rho_{1, k}=\rho_{2, k}\right)\right)$, we compute the average of the terms

$$
\begin{aligned}
& x_{1}=X_{1}\left(\rho_{1,1}, \ldots, \rho_{1, k}, \rho_{1, k+1}, \ldots, \rho_{1, k+(N-k) / 2}\right) \\
& x_{2}=X_{2}\left(\rho_{2,1}, \ldots, \rho_{2, k}, \rho_{2, k+1}, \ldots, \rho_{2, k+(N-k) / 2}\right)
\end{aligned}
$$

and the resulting average, $\frac{x_{1}+x_{2}}{2}$, as the value for the corresponding join ensemble entry $J\left(\rho_{1,1}, \ldots, \rho_{1, k+(N-k) / 2}, \rho_{2, k+1}, \ldots, \rho_{2, k+(N-k) / 2}\right)$.
Note that, since for each one of the $P$ unique combinations selected for the shared pivot parameters, there are $E$ ensemble simulations in both sub-systems, the resulting join ensemble tensor, $\mathcal{J}$, represents $P \times E^{2}$ joined simulations - since, as we saw in the previous subsection, we have $P \times E=B / 2$, this gives us $B^{2} /(4 P)$ simulation entries, (and assuming that $B \gg(4 P)$ ) effectively squaring the simulation density (Figure 6). As we experimentally verify in Section VII, (due to this increased effective density) the decomposition of $\mathcal{J}$ will be a far better approximation for the original system $S$ then the decomposition of the tensor $\mathcal{X}$ which represents the original set of simulations, $X=X_{1} \cup X_{2}$. In fact, the accuracy gains associated with this density increase

- prevents any disadvantages associated with eliminating some of the free parameters, and

- leads to significant overall accuracy gains, even without precise a priori knowledge about parameters to use as pivot and/or values for fixing constants.

2) Zero-Join based Stitching: Note, however, that when $E$ (i.e., sub-system densities) is small, the overall join ensemble density may still be too low to provide accurate analysis. In such a case, we can further boost the overall ensemble density by using zero-join (as opposed to simple join) to stitch the subensembles: when constructing the join ensemble, $J$, for all pairs of simulations in the two sub-ensembles that agree on the parameter values for the $k$ shared parameters (i.e., $\left(\rho_{1,1}=\right.$ $\left.\left.\rho_{2,1}\right) \wedge \ldots \wedge\left(\rho_{1, k}=\rho_{2, k}\right)\right)$, we still compute the average of the terms as described above. But, in this case, if there is a simulation instance,

$$
x_{1}=X_{1}\left(\rho_{1,1}, \ldots, \rho_{1, k}, \rho_{1, k+1}, \ldots, \rho_{1, k+(N-k) / 2}\right)
$$

but the simulation instance

$$
X_{2}\left(\rho_{1,1}, \ldots, \rho_{1, k}, \rho_{2, k+1}, \ldots, \rho_{2, k+(N-k) / 2}\right)
$$

does not exist; then we treat the missing simulation instance as if it exists with 0 value, and we construct the corresponding join ensemble entry $J\left(\rho_{1,1}, \ldots, \rho_{1, k+(N-k) / 2}, \rho_{2, k+1}, \ldots, \rho_{2, k+(N-k) / 2}\right) \quad$ with value $\frac{x_{1}+0}{2}$. We similarly handle simulation instances in $X_{2}$.

Note that zero-joining increases the effective density of the simulation ensemble to $2 \times\left(P \times E^{2}\right) \times E^{2}$, and as we experimentally verify in Section VII, it significantly boosts accuracy in cases where sub-ensemble simulation densities are too low for basic join-based stitching be effective.

\section{Multi-Task Tensor Decomposition (M2TD)}

The difficulty with JE-stitching, of course, is that tensor $\mathcal{J}$ has almost double the number of modes as the tensors $\mathcal{X}_{1}$ and $\mathcal{X}_{2}$. Consequently, its decomposition is likely to be significantly more expensive than the decomposition of these two pre-join tensors. What remains to be shown is that we can, in fact, obtain the decomposition of $\mathcal{J}$ directly from the decompositions of $\mathcal{X}_{1}$ and $\mathcal{X}_{2}$. We discuss this in this section.

Let $\mathcal{X}_{1}$ and $\mathcal{X}_{2}$ be two sub-ensemble tensors corresponding to sub-systems constructed through PF-partitioning of an $N$ parameter system, $S$. Let $J$ be the join ensemble and $\mathcal{J}$ 

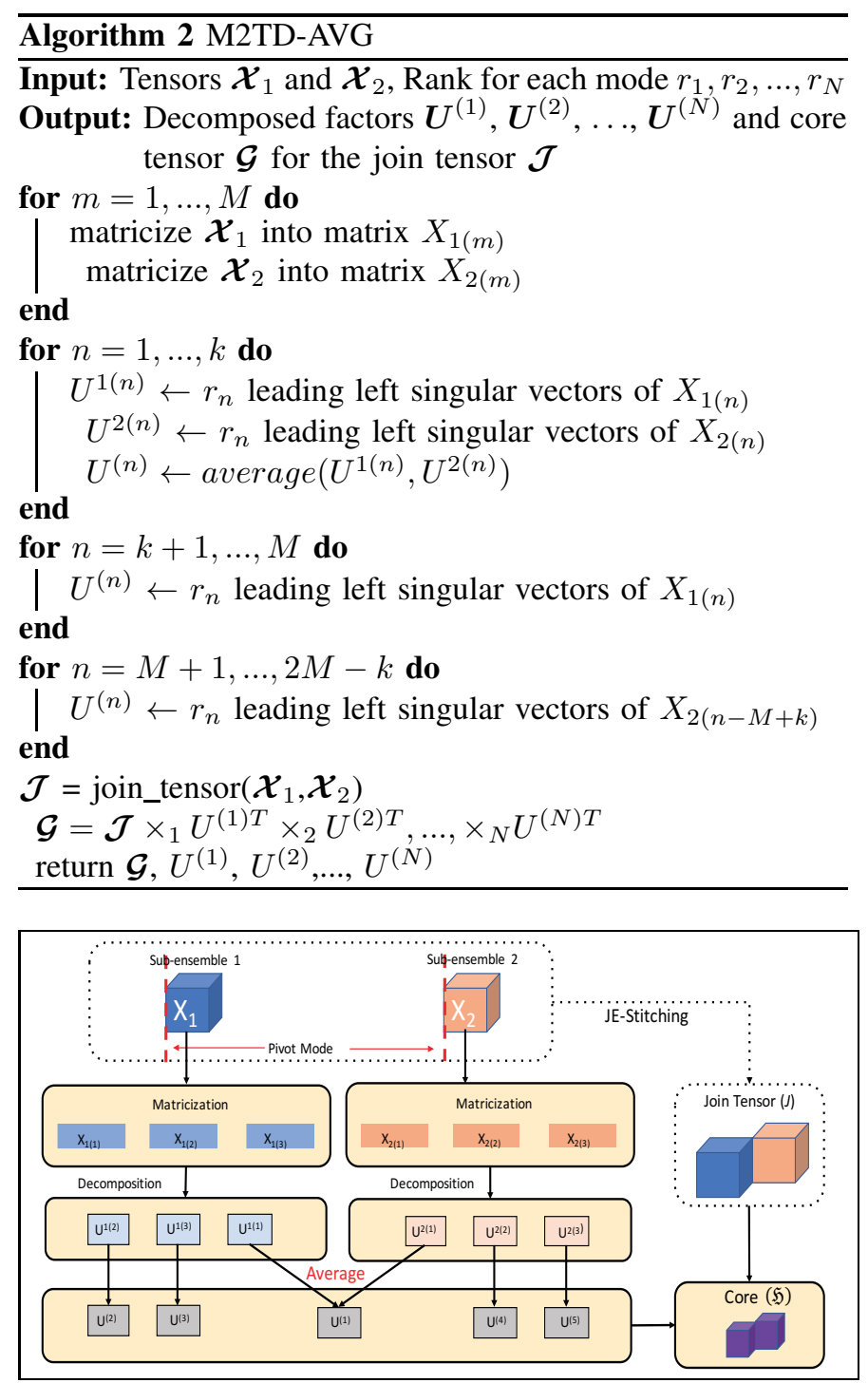

Fig. 7: Overview of M2TD-AVG

be the corresponding join tensor one could obtain through JE-stitching. In this section, we introduce three alternative multi-task tensor decomposition (M2TD) schemes to obtain the decomposition of $\mathcal{J}$ from the decompositions of $\mathcal{X}_{1}$ and $\mathcal{X}_{2}$.

\section{A. M2TD-Average (M2TD-AVG)}

Remember from the earlier sections that both $\mathcal{X}_{1}$ and $\mathcal{X}_{2}$ are $M$-modal tensors, where $M=k+(N-k) / 2$, and that the first $k$ modes are shared. We modify the HOSVD algorithm, presented in Section III-B1, to obtain the proposed M2TDAVG algorithm (Algorithm 2). Intuitively, M2TD-AVG takes the first $k$ factor matrix pairs, $\left(U^{1(n)}, U^{2(n)}\right)$, corresponding to the shared pivot tensors of the independently decomposed tensors, $\mathcal{X}_{1}$ and $\mathcal{X}_{2}$, and averages each pair to obtain a common factor matrix representing both tensors: since factor matrices, $U^{1(n)}$ and $U^{2(n)}$, both map the domain of the corresponding factor to a vector space represented by $r_{n}$ singular factors (sorted in decreasing order of significance),
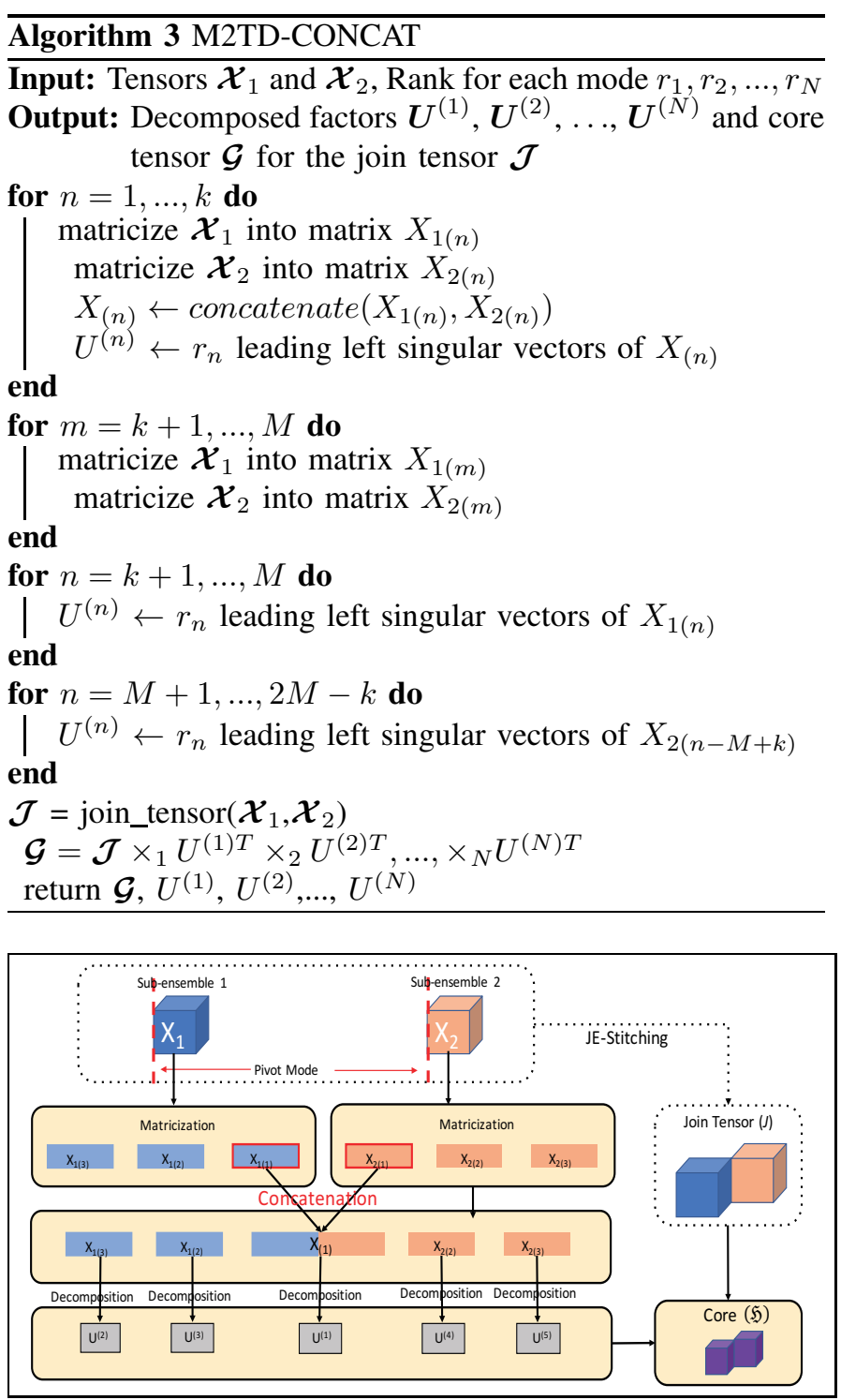

Fig. 8: Overview of M2TD-CONCAT

the operation average $\left(U^{1(n)}, U^{2(n)}\right)$ essentially constructs a new vector space, where each element of the domain is represented by the average vector from the two input vector spaces (Figure 10(a)). Remaining factor matrices are then combined to obtain the core tensor, $\mathcal{G}$ (see Figure 7). As we experimentally verify in Section VII, this leads to a better approximation of the original system than any of the naive ensemble sampling schemes.

\section{B. M2TD-Concatenate (M2TD-CONCAT)}

M2TD-AVG, presented in the previous subsection, recovers the factor matrices for pivot parameters (modes) by averaging the corresponding factor matrices; i.e., by first obtaining the singular vectors of the matricizations and then averaging these singular vectors. However, there is nothing that guarantees that these averages will act as singular vectors themselves.

Instead, the alternative M2TD-CONCAT algorithm (detailed in Algorithm 3 and visualized in Figure 8) avoids this potential 

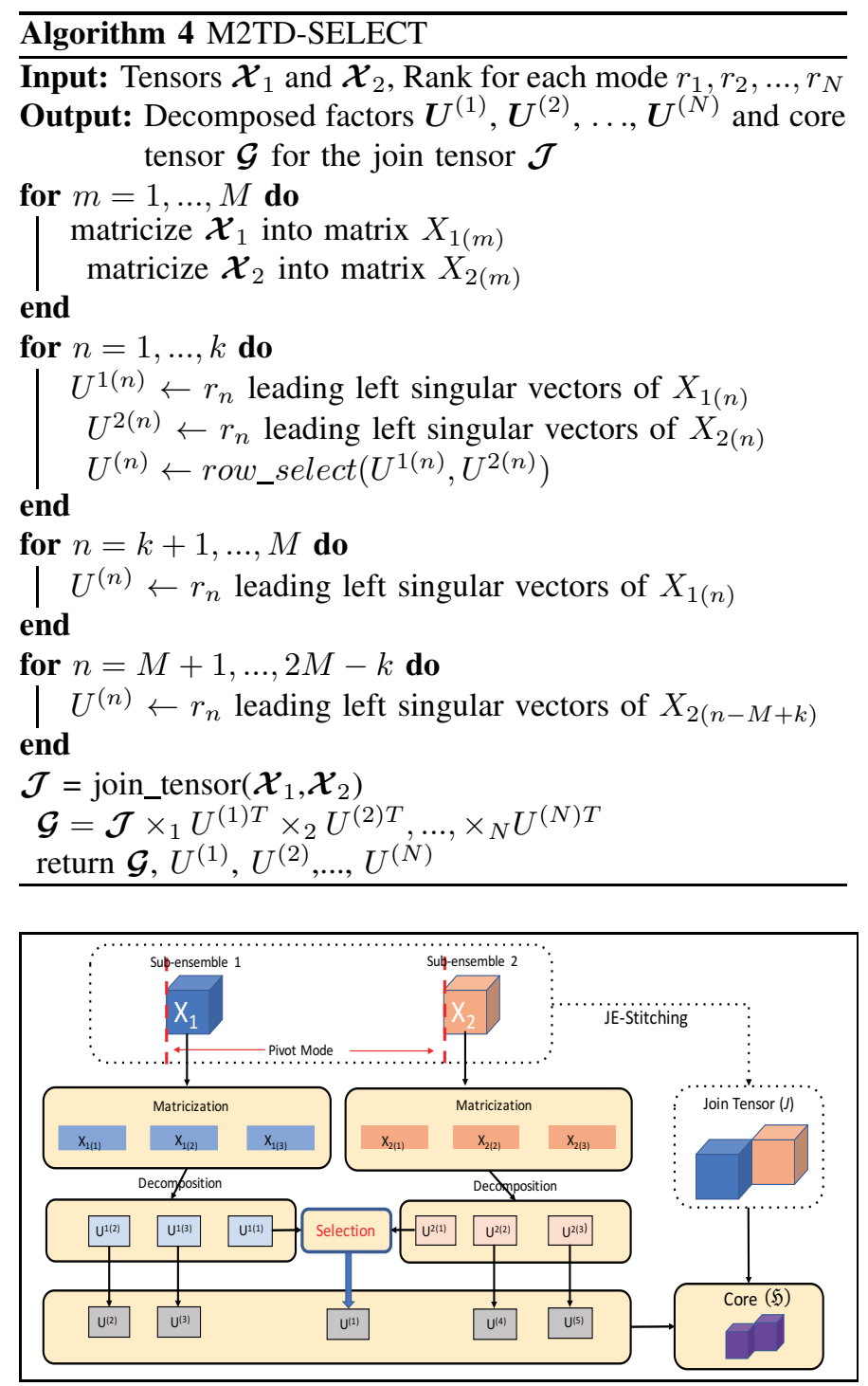

Fig. 9: Overview of M2TD-SELECT

issue by first constructing a concatenated matricization for each pivot mode pair and then seeking the left singular vectors of this combined matricization. Intuitively, M2TD-CONCAT maps the matricizations along the shared/pivot modes back into the higher-modal space and seeks the singular vectors that best represent this higher modal space.

\section{M2TD-Selection (M2TD-SELECT)}

The M2TD-CONCAT algorithm presented above tries to improve the vector averaging scheme of M2TD-AVG through row-by-row concatenation of the pivot matricizations before the corresponding factor matrices are computed. In this subsection, we note that there is an alternative, and potentially more effective, way to improve the M2TD-AVG scheme: once the factor matrices for the pivots are obtained, instead of averaging them, we can carefully select between the individual rows of the corresponding factor matrices and use these selected rows to construct more effective combined factor matrices.

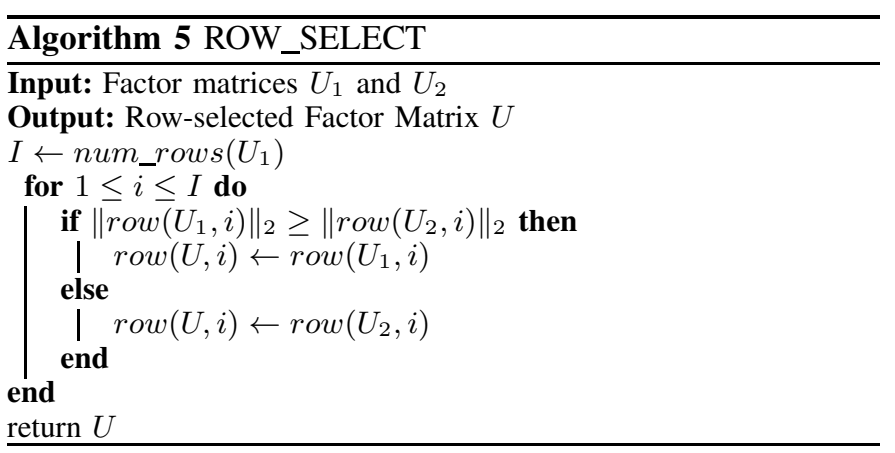

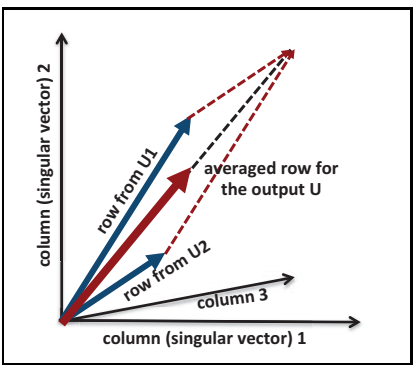

(a) M2TD-AVG

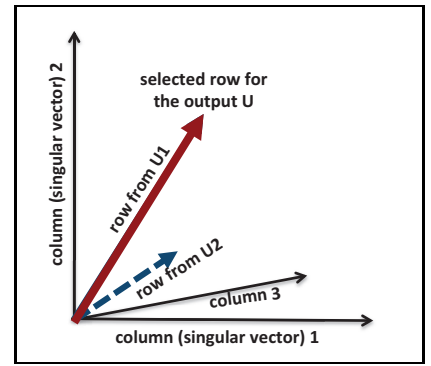

(b) M2TD-SELECT
Fig. 10: Comparison of the row construction processes between M2TD-AVG and M2TD-SELECT

The pseudocode for the process is shown in Algorithm 4 and visualized in Figure 9. Note that the major difference between this algorithm and M2TD-AVG is the line

$$
U^{(n)} \leftarrow \text { row_select }\left(U^{1(n)}, U^{2(n)}\right),
$$

where the factor matrix $U^{(n)}$ is constructed by selecting the appropriate rows from $U^{1(n)}$ or $U^{2(n)}$, instead of simply averaging them. This row selection process is further detailed in Algorithm 5 and visualized in Figure 10(b). As we see here, the key idea is to consider the energies (captured by the 2-norm function) of each row, $i$, in $U_{1}$ and $U_{2}$, and identify which of the two factor matrices provides a higher energy for that particular row. Intuitively, this enables us to identify which of the two factor matrices better represents the entity corresponding to row, $i$, and, given this information, we can construct the row $i$ of the output factor matrix, $U$, by selecting the corresponding row from the factor matrix, $U_{1}$ or $U_{2}$, with a higher representation power for that entity.

As we experimentally verify in Section VII, this selection strategy prevents the row with the lesser energy to act as noise on the description of the corresponding entity and, thus, leads to significantly higher decomposition accuracies. Moreover, as the experiments show, the accuracy gains get higher as we target higher ranking decompositions that maintain more details by seeking a larger number of patterns in the data.

\section{Distributed M2TD (D-M2TD)}

As discussed in Section II, a major challenge with tensor decomposition is its computational and space complexity. This is especially true for the Tucker decomposition with a dense core. In this section, relying on several key properties of the M2TD algorithm, we propose a 3-phase distributed version 


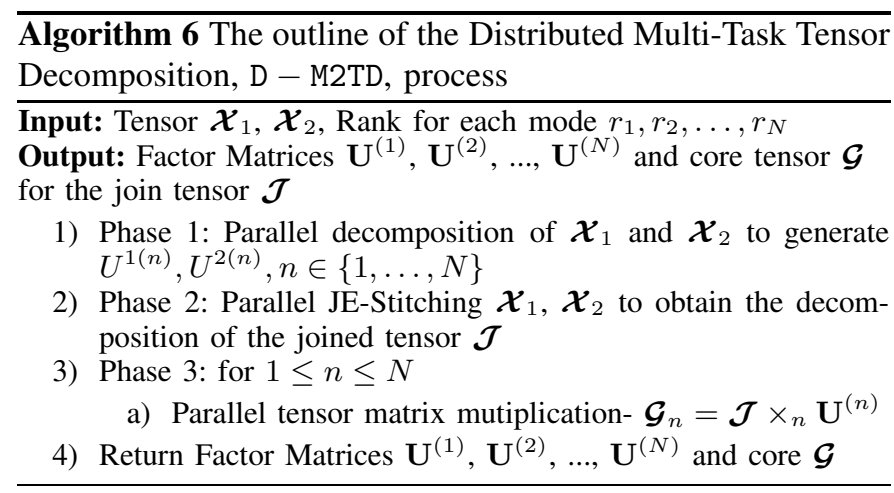

of M2TD that can be efficiently and scalably executed on MapReduce or Spark based platforms (see Algorithm 6):

- Phase 1: Parallel Sub-Tensor Decomposition: Consider the M2TD-SELECT pseudocode in Algorithm 4. Here, $\mathcal{X}_{1}$ and $\mathcal{X}_{2}$ are two sub-tensors corresponding to two sub-systems constructed through PF-partitioning. These low-order subtensors can be decomposed (in parallel) independently from each other. Therefore, this phase can be parallelized using, for example, the popular distributed computing framework, MapReduce, using the following map and reduce operators:

- map: $\left\langle\boldsymbol{\kappa}, \rho_{1}, \rho_{2}, \ldots, \rho_{M}, \mathcal{X}_{\boldsymbol{\kappa}}\left(\rho_{1}, \rho_{2}, \ldots, \rho_{M}\right)\right\rangle$ on $\boldsymbol{\kappa}$. Here, $\kappa$ is the low-order tensor id; i.e., $\kappa \in\{1,2\}$. $\rho_{1}, \rho_{2}, \ldots, \rho_{M}$ together give the coordinate of a cell in the low-order tensor $\mathcal{X}_{\kappa}$. Key-value pairs with the same $\kappa$ are shuffled to the same reducer in the form of $\left\langle k e y: \boldsymbol{\kappa}\right.$, val $\left.: \rho_{1}, \rho_{2}, \ldots, \rho_{M}, \mathcal{X}_{\boldsymbol{\kappa}}\left(\rho_{1}, \rho_{2}, \ldots, \rho_{M}\right)\right\rangle$.

- reduce: $\quad\left\langle k e y: \kappa, \quad\right.$ val $: \rho_{1}, \rho_{2}, \ldots, \rho_{M}$, $\left.\mathcal{X}_{\kappa}\left(\rho_{1}, \rho_{2}, \ldots, \rho_{M}\right)\right\rangle$. The reducer processing the key, $\kappa$, receives the non-zero elements of sub-tensor $\mathcal{X}_{\boldsymbol{\kappa}}$ and decomposes it into sub-factor $U^{\boldsymbol{\kappa}(\boldsymbol{n})}$, where $\boldsymbol{n}$ is the mode id, by using SVD. Finally, reducer appropriately relabels each $U^{\boldsymbol{\kappa}(\boldsymbol{n})}$ as $\boldsymbol{U}^{(\boldsymbol{n})}$ and emits each sub-factor as an independent file, with content $\left\langle k e y: n\right.$, value : $\left.i, j, \boldsymbol{U}^{(\boldsymbol{n})}(i, j)\right\rangle$. Here, $i, j$ are the coordinates of sub-factor $U^{(n)}$.

Note that this step can be further parallelized by leveraging parallel Tucker decomposition techniques, such as [24], [14].

- Phase 2: Parallel JE-Stitching to Obtain Join Tensor, $\mathcal{J}$ : The goal of the stitching process is to increase the effective density of the ensemble. JE-stitching achieves this by joining the two sub-systems along their shared pivot modes to obtain the $\mathcal{J}$ tensor. This process can be parallelized as follows:

- map: $\quad\left\langle\boldsymbol{\kappa}, \rho_{1}, \rho_{2}, \ldots, \rho_{M}, \mathcal{X}_{\boldsymbol{\kappa}}\left(\rho_{1}, \rho_{2}, \ldots, \rho_{M}\right)\right\rangle$. Key-value pairs with the same pivot mode index $\left(\rho_{1}, \rho_{2}, \ldots, \rho_{k}\right)$ are shuffled to the same reducer in the form of $\left\langle k e y:\left(\rho_{1}, \rho_{2}, \ldots, \rho_{k}\right)\right.$, val: $\left.\rho_{1}, \rho_{2}, \ldots, \rho_{M}, \mathcal{X}_{\boldsymbol{\kappa}}\left(\rho_{1}, \rho_{2}, \ldots, \rho_{M}\right)\right\rangle$.

- reduce: $\quad\left\langle k e y:\left(\rho_{1}, \rho_{2}, \ldots, \rho_{k}\right)\right.$, val: $\left.\rho_{1}, \rho_{2}, \ldots, \rho_{M}, \mathcal{X}_{\kappa}\left(\rho_{1}, \rho_{2}, \ldots, \rho_{M}\right)\right\rangle$. The join ensemble $\mathcal{J}\left(\rho_{1}, \rho_{2}, \ldots, \rho_{k}, \ldots\right)$ is constructed for all pairs of $\mathcal{X}_{\kappa}$, that agree on the parameter values for the $k$ pivot parameters.

\begin{tabular}{|l||ccc|}
\hline \multicolumn{1}{|c||}{} & \multicolumn{3}{c|}{ Alternative values } \\
\hline \hline Dynamic systems & Double Pend.; & Triple Pend. & Lorenz System \\
\hline $\begin{array}{l}\text { Parameter resolution } \\
\text { Size of the corresponding } \\
\text { simulation space }(S)\end{array}$ & $60 ;$ & $\mathbf{7 0} ;$ & 80 \\
\hline $\begin{array}{l}\text { Pivot density }(P) \\
\text { Sub-system density }(E)\end{array}$ & $10 \% ;$ & & \\
\hline $\begin{array}{l}\text { Ensemble budget } \\
(B=2 \times P \times E \times S)\end{array}$ & $\left.4 \times 10^{8}\right) ;$ & $70^{5}\left(2 \times 10^{9}\right) ;$ & $80^{5}\left(3 \times 10^{9}\right)$ \\
\hline Target decomposition & $5 \times 10^{5}$, & $\mathbf{1 0 0} \%$ & \\
rank $(r)$ & $5 ;$ & $7 \times 10^{4}$, & $1 \times 10^{5}$, \\
\hline Stitching technique & Join; & $\mathbf{1 0} ;$ & $1 \times 10^{6}$ \\
\hline Number of servers & \multicolumn{3}{|c|}{ Zero-Join } \\
\hline
\end{tabular}

TABLE I: Experiment setup - default values, used unless otherwise specified, are highlighted

- Phase 3: Parallel Tensor-Matrix Multiplication to Recover the Core Tensor: As we see in Section VII, the costliest part of the M2TD algorithm is the final step where the join tensor $\mathcal{J}$ is multiplied by the transposes of the factor matrices to recover the dense core tensor. We parallelize this as follows:
- map: $\left\langle\rho_{1}, \rho_{2}, \ldots, \rho_{N}, \mathcal{J}\left(\rho_{1}, \rho_{2}, \ldots, \rho_{N}\right)\right\rangle$, $\left\langle\boldsymbol{n}, i, j, \boldsymbol{U}^{(n)}(i, j)\right\rangle$. Cells of $\mathcal{J}$ (from Phase 2) with index $\left(\rho_{1}, \rho_{2}, \ldots, \rho_{n-1}, \rho_{n+1}, \ldots, \rho_{N}\right)$ are shuffled to the same reducer in the form of $\left\langle k e y:\left(\rho_{1}, \rho_{2}, \ldots, \rho_{n-1}, \rho_{n+1}, \ldots, \rho_{N}\right)\right.$, val: $\left.\mathcal{J}\left(\rho_{1}, \rho_{2}, \ldots, \rho_{N}\right)\right\rangle$
- map: $\left\langle\boldsymbol{n}, i, j, \boldsymbol{U}^{(n)}(i, j)\right\rangle$. Outputs of Phase $1\left\langle\boldsymbol{n}, i, j, \boldsymbol{U}^{(n)}(i, j)\right\rangle$ are shuffled to the same reducer based on mode id $n$ in the form of $\left\langle k e y:\left(\rho_{1}, \rho_{2}, \ldots, \rho_{n-1}, \rho_{n+1}, \ldots, \rho_{N}\right)\right.$, val: $\left.\boldsymbol{n}, i, j, \boldsymbol{U}^{(n)}(i, j)\right\rangle$

- reduce: The reducer takes

$$
\left\langle k e y:\left(\rho_{1}, . ., \rho_{n-1}, \rho_{n+1}, . ., \rho_{N}\right), \text { val }: \mathcal{J}\left(\rho_{1}, \ldots, \rho_{N}\right)\right\rangle
$$

and

$$
\begin{aligned}
& \left\langle k e y:\left(\rho_{1}, . ., \rho_{n-1}, \rho_{n+1}, . ., \rho_{N}\right), \text { val : } \boldsymbol{n}, i, j, \boldsymbol{U}^{(n)}(i, j)\right\rangle \\
& \text { and performs vector-matrix multiplication } \\
& \text { to emit } \quad\left\langle\left(\rho_{1}, \rho_{2}, \ldots, \rho_{n-1}, j, \rho_{n+1}, \ldots, \rho_{N}\right)\right. \text {, } \\
& \left.\sum_{\rho_{n}=1}^{I_{n}} \mathcal{J}\left(\rho_{1}, \ldots, \rho_{n}, \ldots, \rho_{N}\right) * \boldsymbol{U}^{(n)}\left(\rho_{n}, j\right)\right\rangle .
\end{aligned}
$$

In the next section, we investigate the impact of this parallelization approach to the performance of the proposed partition-stitch sampling through M2TD decomposition.

\section{EXPERIMENTS}

In this section, we report results of the experiments that aim to assess the effectiveness and efficiency of the proposed partition-stitch ensemble sampling strategy and the novel multi-task tensor decomposition (M2TD) scheme. For these experiments, we used the Chameleon cloud platform [1]: we deployed all algorithms on 18 xxlarge instances, with 8-core vCPU, 32GB memory, 160GB disk space. Distributed versions were implemented in Java 8, over Hadoop 2.7.3. The key system parameters and their value ranges are reported in Table I and explained below. 


\section{A. Dynamic Systems}

In these experiments, we consider three dynamic processes: double pendulum, triple pendulum, lorenz system [5]. The code for these systems was obtained from [2] and [3]. These dynamic processes are selected for their varying complexities:

The double pendulum system has four parameters: initial angle, $\phi_{1}$, and weight, $m 1$, of the first pendulum as well as the initial angle, $\phi_{2}$, and weight, $m 2$, of the second pendulum.

The triple pendulum (with variable friction) system is similar, but more complex due to the addition of a third pendulum. Moreover, the system has a different set of initial parameters: the angle $\phi_{1}$ of the first pendulum, the initial angle $\phi_{2}$ of the second pendulum, the initial angle $\phi_{3}$ of the third pendulum, and the friction $f$ of whole system. Intuitively, unlike the double pendulum system, in the triple pendulum system the friction is considered as a simulation parameter.

The Lorenz system is notable for having chaotic solutions for certain initial conditions [5]. The system has four variable parameters: the coordinate of the initial position, $z$, and three other system parameters, $\sigma, \beta, \rho$.

\section{B. Simulation Ensembles}

For the above systems, we construct 5-mode simulation ensembles. Each cell of the 5-mode ensemble simulation tensor encodes the Euclidean distance between the states of the resulting simulated system and the observed system parameters at a given time stamp, for a given quadruple of simulation parameters. Intuitively, each cell encodes the relationship between a given simulation instance to a configuration observed in the real-world.

As we see in Table $\mathrm{I}$, in the experiments, the size of the simulation space varied between $60^{5} \sim 8 \times 10^{8}$ to $80^{5} \sim 3 \times 10^{9}$ simulation instances. In contrast, the simulation instance budgets were on the order of $10^{4}$ to $10^{5}$, indicating that, despite the large number of simulations included in the ensembles, the resulting ensemble tensors were very sparse (densities on the order of $\sim 10^{-4}$ ). Despite this sparsity, for the different configurations considered in Table I, the simulation ensemble required from $25 \mathrm{~GB}$ to $105 \mathrm{~GB}$ data storage.

\section{Alternative Ensemble Construction Schemes}

In this section, we evaluated the M2TD-AVG, -CONCAT, and -SELECT strategies and compared them against the conventional (RANDOM, GRID, and SLICE) ensemble sampling approaches (Section IV). For M2TD-based schemes, we considered the case with a single pivot parameter and, to analyze worst case behavior, we sampled the sub-systems randomly.

\section{Evaluation Criteria}

We compared accuracy and efficiency of alternative schemes, for different target decomposition ranks, different parameter space resolutions, and simulation budgets (see Table I). To measure accuracy, we use the Frobenius norm of the difference tensor (see Section III):

$$
\operatorname{accuracy}(\tilde{\mathcal{X}}, \mathcal{Y})=1-\left(\frac{\|\tilde{\mathcal{X}}-\mathcal{Y}\|}{\|\mathcal{Y}\|_{F}}\right)
$$

\begin{tabular}{|c|c|c|c|c|c|c|c|}
\hline \multicolumn{8}{|c|}{ Accuracy for Double Pendulum System } \\
\hline \multirow[t]{2}{*}{ Res. } & \multirow[t]{2}{*}{ Rank } & \multicolumn{3}{|c|}{ M2TD } & \multirow[t]{2}{*}{ Random } & \multirow[t]{2}{*}{ Grid } & \multirow[t]{2}{*}{ Slice } \\
\hline & & AVG & CONCAT & SELECT & & & \\
\hline \multirow[t]{3}{*}{$\overline{600}$} & $\overline{5}$ & 0.49 & $\overline{0.49}$ & $\overline{0.54}$ & 1E-8 & $\overline{3 \mathrm{E}-4}$ & $\overline{2 \mathrm{E}-4}$ \\
\hline & 10 & 0.50 & 0.50 & 0.62 & $2 \mathrm{E}-7$ & $3 E-4$ & $2 \mathrm{E}-4$ \\
\hline & 20 & 0.52 & 0.53 & 0.56 & $5 \mathrm{E}-6$ & $3 \mathrm{E}-4$ & $2 \mathrm{E}-4$ \\
\hline \multirow[t]{3}{*}{70} & 5 & 0.46 & 0.46 & 0.51 & $7 \mathrm{E}-9$ & $2 \mathrm{E}-4$ & $2 \mathrm{E}-4$ \\
\hline & 10 & 0.47 & 0.48 & 0.57 & $9 \mathrm{E}-8$ & $2 \mathrm{E}-4$ & $2 \mathrm{E}-4$ \\
\hline & 20 & 0.49 & 0.50 & 0.73 & $2 \mathrm{E}-6$ & $2 \mathrm{E}-4$ & $2 \mathrm{E}-4$ \\
\hline \multirow[t]{3}{*}{80} & 50 & 0.46 & 0.46 & 0.50 & $4 \mathrm{E}-9$ & $1 \mathrm{E}-4$ & $1 \mathrm{E}-4$ \\
\hline & 10 & 0.47 & 0.47 & 0.49 & $4 \mathrm{E}-8$ & $1 \mathrm{E}-4$ & $1 \mathrm{E}-4$ \\
\hline & 20 & 0.48 & 0.49 & 0.59 & $1 \mathrm{E}-6$ & $2 \mathrm{E}-4$ & $1 \mathrm{E}-4$ \\
\hline \multicolumn{8}{|c|}{ (a) Accuracy } \\
\hline \multicolumn{8}{|c|}{ Decomposition Time for Double Pendulum System (sec.) } \\
\hline \multirow[t]{2}{*}{ Res. } & Rank & \multicolumn{3}{|c|}{ M2TD } & Random & Grid & Slice \\
\hline & & AVG & CONCAT & SELECT & & & \\
\hline \multirow[t]{3}{*}{ 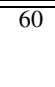 } & $\overline{5}$ & $\overline{808}$ & $\overline{797}$ & $\overline{785}$ & 203 & 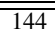 & 167 \\
\hline & 10 & 808 & 819 & 849 & 234 & 148 & 186 \\
\hline & 20 & 1034 & 929 & 935 & 348 & 456 & 258 \\
\hline \multirow[t]{3}{*}{70} & 5 & 1508 & 1581 & 1594 & 312 & 209 & 193 \\
\hline & 10 & 1696 & 1645 & 1576 & 379 & 201 & 244 \\
\hline & 20 & 1866 & 1914 & 1995 & 575 & 744 & 381 \\
\hline \multirow[t]{3}{*}{80} & 5 & 3990 & 3591 & 4907 & 414 & 227 & 336 \\
\hline & 10 & 5232 & 5979 & 6068 & 514 & 239 & 410 \\
\hline & 20 & 5341 & 5707 & 5439 & 860 & 883 & 606 \\
\hline
\end{tabular}

TABLE II: Results for double pendulum system (pivot $=t, P=$ $100 \%, E=100 \%)$

\begin{tabular}{|c|c|c|c|c|c|c|}
\hline \multicolumn{7}{|c|}{ Decomposition Time using Different Numbers of Servers (sec.) } \\
\hline \multirow[t]{2}{*}{ Num. Servers } & \multicolumn{3}{|c|}{ M2TD-SELECT } & \multirow{2}{*}{ Random } & \multirow[t]{2}{*}{$\overline{\text { Grid }}$} & \multirow{2}{*}{ Slice } \\
\hline & Phase 1 & Phase 2 & Phase 3 & & & \\
\hline 2 & $\overline{52}$ & $\overline{817}$ & $\overline{4167}$ & $\overline{670}$ & $\overline{420}$ & $\overline{488}$ \\
\hline 6 & 62 & 383 & 1802 & $\overline{464}$ & 275 & 318 \\
\hline 10 & 61 & 371 & 1318 & 415 & 237 & 280 \\
\hline 14 & 65 & 354 & 1279 & 381 & 214 & 253 \\
\hline 18 & 67 & 363 & 1118 & 379 & 201 & 244 \\
\hline
\end{tabular}

TABLE III: Different number of servers (Double pendulum, resolution=70, rank $=10$, pivot $=t, P=100 \%, E=100 \%$ )

where $\tilde{\mathcal{X}}$ is the reconstructed tensor (after sampling and decomposition), while $\mathcal{Y}$ is the tensor corresponding to the full simulation space. We also report the decomposition times.

\section{E. Discussions of the Results}

1) General Overview: Table II focuses on the double pendulum system and compares accuracies and decomposition times for various approaches considered in this paper for the different target ranks and for different parameter resolutions. As we see in the table, the M2TD-based algorithms provide several orders better accuracy than the conventional approaches, with the same number of simulation instances. As expected, among the conventional schemes, the Random strategy provides the worst and the Grid strategy provides the best accuracy; however, even Grid is $\sim 1000 \times$ worse than the proposed M2TD-SELECT algorithm. As also expected, among the M2TD-based algorithms, M2TD-SELECT provides the best overall accuracy: moreover, the relative performance gains of M2TD-SELECT algorithm further increases for larger decomposition ranks, indicating that as we seek more detailed patterns in the ensemble, M2TD-SELECT better captures these underlying patterns in the data.

In the Table, we also see that M2TD-based algorithms are somewhat more expensive than the conventional sampling strategies; but the gains in accuracy are several orders higher 


\begin{tabular}{|c|c|c|c|c|c|c|}
\hline \multicolumn{7}{|c|}{ Accuracy for Different Systems } \\
\hline \multirow[t]{2}{*}{ Dyn.System } & \multicolumn{3}{|c|}{ M2TD } & \multirow[t]{2}{*}{ Random } & \multirow[t]{2}{*}{ Grid } & \multirow[t]{2}{*}{ Slice } \\
\hline & AVG & CONCAT & SELECT & & & \\
\hline$\overline{\text { D.P. }}$ & 0.47 & 0.48 & 0.57 & $9 \mathrm{E}-8$ & $2 \mathrm{E}-4$ & $2 \mathrm{E}-4$ \\
\hline T.P. & 0.25 & 0.25 & 0.31 & $6 \mathrm{E}-8$ & $2 \mathrm{E}-4$ & $1 \mathrm{E}-4$ \\
\hline L.S. & 0.31 & 0.32 & 0.36 & $4 \mathrm{E}-8$ & $2 \mathrm{E}-4$ & $1 \mathrm{E}-4$ \\
\hline \multicolumn{7}{|c|}{ (a) Accuracy } \\
\hline \multicolumn{7}{|c|}{ Decomposition Time for Different Systems (sec.) } \\
\hline \multirow[t]{2}{*}{ Dyn.System } & \multicolumn{3}{|c|}{ M2TD } & Random & \multirow[t]{2}{*}{ Grid } & \multirow[t]{2}{*}{ Slice } \\
\hline & AVG & CONCAT & SELECT & & & \\
\hline$\overline{\text { D.P. }}$ & $\overline{1696}$ & $\overline{1645}$ & $\overline{1576}$ & $\overline{\overline{379}}$ & 201 & 244 \\
\hline T.P. & 992 & 1422 & 1106 & 221 & 180 & 166 \\
\hline L.S. & 1728 & 1850 & 1705 & 444 & 230 & 211 \\
\hline
\end{tabular}

TABLE IV: Results for different dynamical systems (resolution=70, rank $=10$, pivot $=t, P=100 \%, E=100 \%$ )

\begin{tabular}{|c||ccc|ccc|}
\hline \multicolumn{7}{|c|}{ Accuracy for Different Ensemble Budgets $(B)$} \\
\hline Budget & AVG & CONCAT & SEL. & & & \\
& $3.5 \mathrm{E}-5$ & $3.4 \mathrm{E}-5$ & $\mathbf{4 . 1 E}-5$ & $9 \mathrm{E}-9$ & $2 \mathrm{E}-5$ & $2 \mathrm{E}-6$ \\
\hline \hline $4 \times 10^{4}$ (join) & $3.3 \mathrm{E}-3$ & $3.2 \mathrm{E}-3$ & $\mathbf{3 . 9 E}-3$ & $9 \mathrm{E}-9$ & $2 \mathrm{E}-5$ & $2 \mathrm{E}-6$ \\
$4 \times 10^{4}$ (zero-join) & 0.47 & 0.48 & $\mathbf{0 . 5 7}$ & $9 \mathrm{E}-8$ & $2 \mathrm{E}-4$ & $2 \mathrm{E}-4$ \\
$4 \times 10^{5}$ & \multicolumn{7}{|c|}{ (a) Accuracy } \\
\hline
\end{tabular}

\begin{tabular}{|c||ccc|ccc|}
\hline \multicolumn{7}{|c|}{ Decomposition Time for Different Ensemble Budgets $(B)$ (sec.) } \\
\hline Budget & AVG & CONCAT & SEL. & & & \\
& 200 & 201 & 200 & 190 & 175 & 183 \\
\hline \hline $4 \times 10^{4}$ (join) & 596 & 598 & 592 & 190 & 175 & 183 \\
$4 \times 10^{4}$ (zero-join) & 1696 & 1645 & 1576 & 379 & 201 & 244 \\
$4 \times 10^{5}$ & & & & & \\
\hline
\end{tabular}

(b) Time (sec.)

TABLE V: Results for different ensemble budgets (Double pendulum, resolution $=70$, rank $=10$, pivot $=t$; note that $B=$ $4 \times 10^{5}$ corresponds to the case where both pivot, $P$, and sub-systems, $E$, have $100 \%$ densities)

than the decomposition time overheads of M2TD-based techniques. This is because, as highlighted in Section V-C, the proposed partition-stitch technique increases the effective density of the join ensemble. Consequently, the increase in the decomposition is well amortized by the increase in the effective simulation density. In these experiments, each double pendulum simulation took roughly $0.66 \mathrm{~ms}$. Given this, obtaining an ensemble simulation with density $70^{4}\left(=70^{2} \times 70^{2}\right)$ would require roughly 16000 seconds (ignoring the additional time to decompose). In contrast, the proposed M2TD based techniques are able to achieve the same effective density by running only $2 \times 70^{2}$ simulations in just 46 seconds and obtain the ensemble decomposition in an additional $\sim 1600$ seconds. This points to the impressive performance gains provided by the proposed multi-task tensor decomposition (M2TD) technique.

One question that remains is whether we could have joined the sub-ensembles directly into tensor $\mathcal{J}$ to decompose instead of relying on the M2TD techniques: the answer to this question is a strong no: for the experiments reported in Table II, with the configuration of 18 xxlarge servers, direct decomposition of the resulting dense tensor was not feasible due to memory limitations.

2) Decomposition Time Distribution: Table III presents how the decomposition time is split among the three phases of the map-reduce process described in Section VI-D. The table also shows how the execution time varies as we change the

\begin{tabular}{|c|c|c|c|c|c|c|}
\hline \multicolumn{7}{|c|}{ Accuracy for Different Pivot Densities $(P)$} \\
\hline \multirow{2}{*}{ P. Density } & \multicolumn{3}{|c|}{ M2TD } & \multirow{2}{*}{\multicolumn{2}{|c|}{ Random Grid }} & \multirow[t]{2}{*}{ Slice } \\
\hline & AVG & CONCAT & SELECT & & & \\
\hline $10 \%$ & $3.5 \mathrm{E}-2$ & $7.6 \mathrm{E}-3$ & $3.6 \mathrm{E}-2$ & $9 \mathrm{E}-9$ & $2 \mathrm{E}-5$ & $2 \mathrm{E}-6$ \\
\hline $100 \%$ & 0.47 & 0.48 & 0.57 & $9 \mathrm{E}-8$ & $2 \mathrm{E}-4$ & $2 \mathrm{E}-4$ \\
\hline \multicolumn{7}{|c|}{ (a) Accuracy } \\
\hline \multicolumn{7}{|c|}{ Decomposition Time for Different Pivot Densities $(P)$ (sec.) } \\
\hline \multirow[t]{2}{*}{ P.Density } & \multicolumn{3}{|c|}{ M2TD } & Random & Grid & Slice \\
\hline & AVG & CONCAT & SELECT & & & \\
\hline $10 \%$ & $\overline{\overline{606}}$ & $\overline{597}$ & $\overline{\overline{607}}$ & $\overline{190}$ & 175 & $\overline{183}$ \\
\hline $100 \%$ & 1696 & 1645 & 1576 & 379 & 201 & 244 \\
\hline
\end{tabular}

TABLE VI: Results for different pivot densities (Double pendulum, resolution $=70$, rank $=10$, pivot $=t, E=100 \%$ )

\begin{tabular}{|c|c|c|c|c|c|c|}
\hline \multicolumn{7}{|c|}{ Accuracy for Different Sub-system Densities $(E)$} \\
\hline E. Density & \multicolumn{3}{|c|}{ M2TD } & \multicolumn{2}{|c|}{ Random Grid } & Slice \\
\hline $10 \%($ join $)$ & $4 \mathrm{E}-5$ & $4 \mathrm{E}-5$ & 4.5E-5 & 9E-9 & $2 \mathrm{E}-5$ & $2 \mathrm{E}-6$ \\
\hline $10 \%$ (zero-join) & $3.4 \mathrm{E}-3$ & $3.3 \mathrm{E}-3$ & 3.8E-3 & 9E-9 & $2 \mathrm{E}-5$ & $2 \mathrm{E}-6$ \\
\hline $100 \%$ & 0.47 & 0.48 & 0.57 & 9E-8 & $2 \mathrm{E}-4$ & $2 \mathrm{E}-4$ \\
\hline \multicolumn{7}{|c|}{ (a) Accuracy } \\
\hline \multicolumn{7}{|c|}{ Decomposition Time for Different Sub-system Densities $(E)$ (sec.) } \\
\hline \multirow[t]{2}{*}{ E. Density } & \multicolumn{3}{|c|}{ M2TD } & \multirow[t]{2}{*}{ Random } & \multirow[t]{2}{*}{ Grid } & \multirow[t]{2}{*}{ Slice } \\
\hline & AVG & CONCAT & SELECT & & & \\
\hline$\overline{10 \%(\text { join })}$ & $\overline{207}$ & $\overline{202}$ & $\overline{201}$ & $\overline{190}$ & $\overline{175}$ & 183 \\
\hline $10 \%$ (zero-join) & 602 & 640 & 617 & 190 & 175 & 183 \\
\hline $100 \%$ & 1696 & 1645 & 1576 & 379 & 201 & 244 \\
\hline
\end{tabular}

TABLE VII: Results for different sub-system densities (Double pendulum, resolution=70, rank $=10$, pivot $=t, P=100 \%$ )

number of servers allocated for the decomposition process. As we see in this table, as expected, the third phase where we recover the core tensor of the decomposition is the costliest step of the process. We also see that allocating more servers indeed helps bring the cost of this phase down; however, there are diminishing returns due to data communication overheads.

3) Varying Data Sets: In Table IV, we study the accuracy and decomposition time results for different dynamic systems. As we see here, also for the triple pendulum and Lorenz systems, we observe the very same pattern: M2TD-SELECT provides the best accuracy among all alternatives, providing several orders of magnitude gain in accuracy relative to the conventional schemes.

4) Varying Budgets and Zero-Joins: In the default experiments considered above, the budget was selected such that the sub-ensembles would have a perfect density of 1.0. In the first row of Table $\mathrm{V}$, we reduced the ensemble budget by taking $1 / 10^{t h}$ of the samples we considered in the previous examples. Naturally, this results in a drop in accuracy for all approaches. However, M2TD-based schemes remain several orders better than the conventional approaches.

The table also shows that when the budgets (thus subensemble densities) are low, we can boost the overall accuracy by leveraging zero-joins (introduced in Section V-C), rather than using simple joins when implementing JE-stitching.

5) Varying Pivot/Sub-Ensemble Densities: Tables VI and VII show the impact of reduced pivot and sub-ensemble densities (i.e., $P$ and $E$ ) respectively. As we see here, the overall pattern is as before: reduction in the simulation budget 


\begin{tabular}{|c|c|c|c|c|c|c|}
\hline \multicolumn{7}{|c|}{ Accuracy for Different Pivot Parameters } \\
\hline \multirow[t]{2}{*}{ Pivot } & \multicolumn{3}{|c|}{ M2TD } & \multirow{2}{*}{\multicolumn{3}{|c|}{ Random $\quad$ Grid $\quad$ Slice }} \\
\hline & AVG & CONCAT & SELECT & & & \\
\hline $\bar{t}$ & $\overline{0.47}$ & $\overline{0.48}$ & $\overline{0.57}$ & \multirow[t]{5}{*}{$\overline{9 \mathrm{E}-8}$} & \multirow[t]{5}{*}{$2 \mathrm{E}-4$} & \multirow[t]{5}{*}{$2 \mathrm{E}-4$} \\
\hline$\phi_{1}$ & 0.35 & 0.36 & 0.40 & & & \\
\hline$\phi_{2}$ & 0.40 & 0.41 & 0.56 & & & \\
\hline$m_{1}$ & 0.58 & 0.59 & 0.71 & & & \\
\hline$m m_{2}$ & 0.41 & 0.40 & 0.42 & & & \\
\hline \multicolumn{7}{|c|}{ (a) Accuracy } \\
\hline \multicolumn{7}{|c|}{ Decomposition Time for Different Pivot Parameters (sec.) } \\
\hline \multirow[t]{2}{*}{ Pivot } & \multicolumn{3}{|c|}{ M2TD } & \multirow[t]{2}{*}{ Random } & \multirow[t]{2}{*}{ Grid } & \multirow[t]{2}{*}{ Slice } \\
\hline & AVG & CONCAT & SELECT & & & \\
\hline $\bar{t}$ & 1696 & $\overline{1645}$ & 1576 & \multirow[t]{5}{*}{$\overline{\overline{379}}$} & \multirow[t]{5}{*}{201} & \multirow[t]{5}{*}{244} \\
\hline$\phi_{1}$ & 1607 & 1673 & 1673 & & & \\
\hline$\phi_{2}$ & 1694 & 1677 & 1571 & & & \\
\hline$m_{1}$ & 1661 & 1512 & 1697 & & & \\
\hline$m_{2}$ & 1556 & 1602 & 1538 & & & \\
\hline
\end{tabular}

TABLE VIII: Results for different pivots (Double pendulum, resolution=70, rank $=10, P=100 \%, E=100 \%$; 3-mode sub-systems are created in such a way that free parameters of the same pendulum are kept in the same sub-system)

reduces the overall accuracy; however, M2TD-based schemes provide significantly higher accuracy overall.

An interesting observation, however, is that (while the total number of simulations is the same) reduction in the pivot subensemble density has a significantly higher impact than the reduction in the pivot density: this is because, as discussed in Section V-C, the effective density of a stitched simulation ensemble is proportional to $P \times E^{2}$, and thus reductions in $E$ have a more significant impact than reductions in $P$ : this further confirms our initial hypothesis that maintaining subensemble densities high is important for accurate characterization of the system being studied.

6) Selection of the Pivot Parameter: In Table VIII, we vary the pivot parameter ${ }^{1}$ : as we expected, which parameter is selected as the pivot has some impact on the accuracy of the proposed partition-stitch scheme. However, whichever pivot is selected, the overall accuracy is several orders of magnitude better than that of conventional schemes, indicating that we do not need very precise information about the system being studied to decide how to partition the system.

\section{CONCLUSIONS}

In this paper, we presented a tensor-based framework to represent and analyze large simulation ensembles to support decision making in the presence of complex, dynamic systems. Noting that simulation ensembles and the corresponding tensors are often extremely sparse due to the size of the potential simulation parameter space, we proposed a partition-stitch sampling scheme to help increase the effective density of the simulation samples to boost accuracy. We have complemented this sampling scheme with a novel Multi-Task Tensor Decomposition (M2TD) to efficiently stitch these sub-ensembles in a way that leverages partial and imperfect knowledge from partial dynamical systems to effectively obtain a global

\footnotetext{
${ }^{1}$ Due to space constraints, we omit experiments where we keep the same pivot parameter, but vary the groupings of free parameters. The results are similar to the results of pivot parameter selection.
}

view of the complex process being simulated. Experiment results showed the efficiency and the effectiveness of the proposed approach relative to more conventional techniques for constructing simulation ensembles.

\section{REFERENCES}

[1] Chameleon Cloud. 2017.

[2] Code for double and triple pendulum systems, http://www.ita.uniheidelberg.de/ chfeder/applets.shtml, 2017.

[3] Code for the Lorenz system, http://www.algosome.com/articles/lorenzattractor-programming-code.html, 2017.

[4] Committee on Environment and Natural Resources, Grand Challenges for Disaster Reduction, National Science and Tech. Council, 2008.

[5] P. Berg et al. Order within Chaos: Towards a Deterministic Approach to Turbulence. 1984.

[6] STEM. The spatiotemporal epidemiological modeler project. http://www.eclipse.org/stem/ , 2017.

[7] C. A. Andersson and R. Bro. The N-Way Toolbox for Matlab. Chem. and Intel. Lab. Systems, 52(1):1-4, 2000.

[8] B. W. Bader, T. G. Kolda, et al. MATLAB Tensor Toolbox Version 2.5, Available online, January 2012.

[9] C. H. Chen, L. H. Lee, Stochastic Simulation Optimization: An Optimal Computing Budget Allocation, World scientific, 2010.

[10] R. Fisher. The Design of Experiments, 1935.

[11] R. A. Harshman, Foundations of the PARAFAC procedure: Model and conditions for an explanatory multi-mode factor analysis. UCLA Working Papers in Phonetics, 16:1-84, 1970.

[12] S. Huang, K. S. Candan, M. L. Sapino. BICP: Block-Incremental CP Decomposition with Update Sensitive Refinement. CIKM 2016

[13] B. Jeon, Inah Jeon, Lee Sael and U Kang SCouT: Scalable Coupled Matrix-Tensor Factorization - Algorithm and Discoveries. ICDE 2016.

[14] I. Jeon, E. Papalexakis, U. Kang, and C. Faloutsos. HaTen2: Billionscale tensor decompositions. ICDE 2015

[15] N.L. Johnson. Sequential analysis: a survey. Journal of the Royal Statistical Society, Series A. Vol. 124 (3), 372411, 1961.

[16] U. Kang, et al. Gigatensor: scaling tensor analysis up by 100 times algorithms and discoveries. KDD 2012.

[17] M. Kim and K.S. Candan. Decomposition by normalization (DBN): leveraging approximate functional dependencies for efficient $\mathrm{CP}$ and tucker decompositions. Data Min. Knowl. Discov. 30(1): 1-46, 2016.

[18] S. Klus, P. Gel $\beta$, S. Peitz, C. Schutte, Tensor-based dynamic mode decomposition, ArXiv, 2017.

[19] T. G. Kolda, J. Sun. Scalable tensor decompositions for multi-aspect data mining. ICDM 2008.

[20] T.G. Kolda and B.W. Bader. Tensor Decompositions and Applications. SIAM Rev. 51, 3, 455-500. 2009.

[21] X.Li, S.Huang, K.Candan and M.Sapino, Focusing Decomposition Accuracy by Personalizing Tensor Decomposition (PTD). CIKM'14.

[22] X. Li, S.Y. Huang, K.S. Candan and M.L. Sapino, 2PCP: Two-phase CP decomposition for billion-scale dense tensors. ICDE 2016.

[23] Sicong Liu, et al. Notes2: Networks-of-traces for epidemic spread simulations. AAAI Workshop on Computational Sustainability, 2015.

[24] E. Papalexakis, C. Faloutsos, and N. Sidiropoulos. Parcube: Sparse parallelizable tensor decompositions. PKDD,2012.

[25] G. Pedrielli, et al. Single-run Simulation Optimization Through Time Dilation and Optimal Computing Budget Allocation. Stochastic Models of Manufacturing and Service Operation, 2015.

[26] G. Pedrielli, et al. Empirical analysis of the performance of variance estimators in sequential single-run ranking selection: the case of time dilation algorithm. WSC'16

[27] A.H. Phan and A. Cichocki, PARAFAC algorithms for large-scale problems, Neurocomputing, vol. 74, no. 11, pp. 1970-1984, 2011.

[28] S. Poccia et al. SIMDMS: Data Management and Analysis to Support Decision Making through Large Simulation Ensembles. EDBT 2017.

[29] M.Rogers, L. Li, S. Russell, Multilinear Dynamical Systems for Tensor Time Series. NIPS 2003.

[30] L.W.Schruben, et al. Simulation Optimization Using Simultaneous Replications and Event Time Dilation, WSC 1997.

[31] C. E. Tsourakakis, Mach: Fast randomized tensor decompositions. Arxiv :0909.4969, 2009

[32] L. Tucker, Some mathematical notes on three-mode factor analysis. Psychometrika, 31:279-311, 1966. 